\title{
Review Article \\ Pathogenicity of Virulent Species of Group C Streptococci in Human
}

\author{
Marta Kłos and Jadwiga Wójkowska-Mach \\ Department of Microbiology, Jagiellonian University, Czysta 18, 31-121 Kraków, Poland \\ Correspondence should be addressed to Marta Kłos; klosmartha@gmail.com
}

Received 13 February 2017; Revised 24 April 2017; Accepted 21 May 2017; Published 12 June 2017

Academic Editor: Nahuel Fittipaldi

Copyright (C) 2017 Marta Kłos and Jadwiga Wójkowska-Mach. This is an open access article distributed under the Creative Commons Attribution License, which permits unrestricted use, distribution, and reproduction in any medium, provided the original work is properly cited.

\begin{abstract}
Group C streptococci (GCS) are livestock pathogens and they often cause zoonotic diseases in humans. They are Gram-positive, in mostly $\beta$-hemolytic and facultative anaerobes. Because of their close evolutionary kinship with group A streptococci (GAS), GCS share many common virulence factors with GAS and cause a similar range of diseases. Due to the exchange of genetic material with GAS, GCS belong to bacteria that are difficult to be distinguished from group A streptococci; GCS are often treated in microbiological diagnostics as contamination of the culture. This report focuses mainly on the pathogenicity of virulent species of GCS and their association with human diseases. The condition that is most frequently quoted is pharyngitis. In this paper, the virulence factors have also been mentioned and an interesting link has been made between GCS and the pathogenesis of rheumatic diseases among the native people of India and Aboriginal populations.
\end{abstract}

\section{Introduction}

Group C streptococci, according to Lancefield's work of 1933, belong to Gram-positive bacteria, catalase-negative and facultative anaerobes [1-4]. The division of streptococci into groups was made by Lancefield; the research concerned 106 strains of Streptococcus haemolyticus isolated from many sources (humans, animals, and dairy products) and was based on the carbohydrate $\mathrm{C}$ expressed in the wall of bacteria [4]. Using sera, agglutination reactions, and reactions of precipitation Lancefield classified the strains into respective groups [4]. Based on the results of the anti-C precipitin test, it was stated that the strains of group $\mathrm{C}$ came from various sources other than human and numbered 49 strains. Those strains of group C can hardly, or impossibly, be distinguished from human hemolytic streptococci [4].

GCS identification can be difficult due to their close kinship with group A streptococci-they have a common evolutionary origin, and they are well-known for their exchange of genetic material, share many of their virulence genes, and cause similar diseases [5]. The study of the epidemiology of human GCS infections has recently been made possible mainly owing to the development of modern methods of molecular diagnosis. GCS are mainly livestock commensals or pathogens, although they can also be the part of the human flora of the nose, throat, intestines, or skin $[3,6]$. The main origin of virulent species of GCS that cause human diseases is animals, unpasteurized milk, and unpasteurized dairy products [2, 6-10]. Infections caused by streptococci can be invasive or noninvasive, and they cannot be distinguished merely on the basis of their clinical presentation [3].

Astete reports that Streptococcus equi is regarded as the immediate antecedent of group C streptococci and is also the cause of almost $2 \%$ of all infections caused by GCS [1]. Table 1 depicts the species currently belonging to GCS (Table 1). According to Facklam GCS contains four species: S. dysgalactiae subs. dysgalactiae, S. dysgalactiae subs. equisimilis, S. equi subs. equi, S. equi subs. zooepidemicus, and S. constellatus subs. pharyngis [11]. The streptococcus of group C, which most often causes infection in humans, is Streptococcus dysgalactiae subs. equisimilis (SDSE) [10, 12]; it may cause skin infections (cellulitis) and recurrent blood infections [13]. According to Clarke the GCS species, not mentioned before, most often isolated from the human throat is $S$. milleri [8], also known as S. anginosus group [11]. 
TABLE 1: Phenotypic characteristics of group C streptococci.

\begin{tabular}{|c|c|c|c|c|c|c|c|}
\hline Species & Lancefield group & $\beta$-hemolysis & PYR test & $\mathrm{Sbl}^{\mathrm{a}}$ & $\operatorname{Tre}^{\mathrm{b}}$ & Origin & References \\
\hline $\begin{array}{l}\text { S. dysgalactiae } \\
\text { subs. } \\
\text { dysgalactiae }\end{array}$ & $\mathrm{C}$ & - & - & $\mathrm{v}$ & + & Animals & [11] \\
\hline $\begin{array}{l}\text { S. dysgalactiae } \\
\text { subs. equisimilis }\end{array}$ & A, C, G & + & - & - & + & Human, animals & {$[2,5,7,10-12,14]$} \\
\hline S. equi subs. equi & $\mathrm{C}$ & + & - & - & - & Animals & [11] \\
\hline $\begin{array}{l}\text { S. equi subs. } \\
\text { zooepidemicus }\end{array}$ & $\mathrm{C}$ & + & - & + & $\mathrm{v}$ & Human, animals, unpasteurized dairy products & {$[2,11]$} \\
\hline $\begin{array}{l}\text { S. constellatus } \\
\text { subs. pharyngis }\end{array}$ & $\mathrm{C}$ & + & - & - & + & Human & [11] \\
\hline
\end{tabular}

\section{Identification}

In contemporary laboratories the identification according to the carbohydrate group GCS strains may be conducted by means of latex agglutination [5, 13]. Aside from the classification according to the carbohydrate group, there are many other identification methods, which will allow us to discriminate species within GCS and to discriminate GCS from other streptococci. Some selected phenotypic features of the GCS species are shown in Table 1 (Table 1).

Phenotypes of group C streptococci and group G streptococci (GGS) form a large colony, which makes them different from the phenotypes belonging to the $S$. anginosus group, which form a small colony, irrespective of the carbohydrate group [7]. The aforementioned S. anginosus group (called $S$. milleri by some authors) contains three species: $S$. anginosus, $S$. constellatus, and $S$. intermedius $[7,8,11,15]$. The species that belong to the $S$. anginosus group contain in their cellular wall the carbohydrate groups A, C, F, and G or they contain none [11]. GCS and S. anginosus group can be identified on the basis of $\beta$-hemolytic activity [5]. All the species of the $S$. anginosus group and almost all the phenotypes of GCS cause beta-hemolysis except $S$. dysgalactiae subs. dysgalactiae $[9,11]$. In order to discriminate GCS or GGS from GAS the PYR test is applied (the L-pyroglutamyl amino-peptidase test) [5]. The PYR test shows a positive result only in the case of GAS; both in the case of GCS and in the $S$. anginosus group no positive reaction had been found $[5,11]$.

In Angeletti's work we find two reliable optochin sensitivity and bile solubility tests, the tests mentioned also in Facklam's observation $[11,16]$. Facklam shows that virulent streptococci, including those from group C, manifest a negative reaction in optochin sensitivity and bile solubility tests [11]. Aside from the aforementioned methods, Clarke et al. report that GCS can also be differentiated from other species through their capacity for sorbitol and trehalose fermentation ([8], Table 1). Among the phenotypes belonging to GCS, only S. equi subs. zooepidemicus can ferment sorbitol, whereas S. dysgalactiae subs. dysgalactiae manifests variable reactions $[2,11]$. Almost all the phenotypes of GCS, except S. equi subs. equi and S. equi subs. zooepidemicus, which manifests a variable response, ferment trehalose [11]. It is interesting to note that none out of the 49 strains attributed in 1933 by Lancefield to group C fermented trehalose [4].

One can include within the molecular techniques of streptococci identification the emm typing, MALDI-TOF MS method (Matrix-Assisted Laser Desorption/Ionization Timeof-Flight Mass Spectrometry), and the sequencing of selected genes. The examination of the presence of emm and emmlike genes, called emm typing, allows us to determine the $\mathrm{M}$ protein serotypes [17]. In Dinkla's research the emm types were identified by means of PCR analysis, with the use of starters recommended by the Center of Disease Control [18]. In the case of GAS the emm gene sequence technique is combined with T typing, a fact that is not possible in GCS or GGS, for GCS/GGS contain no T proteins [17].

MALDI-TOF MS identification is based on the protein composition of cell bacteria, especially ribosomal proteins. In Angeletti's tests 158 strains have been isolated, all of them belonging to the viridans group streptococci (VGS), and examined in the MALDI-TOF MS; then genes $t u f$, $\operatorname{sod} A$, and $r p o B$ were amplified and sequenced [16]. It has been stated that in Angeletti MALDI-TOF MS sensitivity obtained 100\% in comparison with the phenotypical tests [16]. To identify S. dysgalactiae subs. equisimilis strains Ciszewski presents the RISA method (analysis of ribosomal intergenic region) [19]. In the RISA method, in the course of PCR reaction, one uses S. dysgalactiae species-specific primers and SDSE subspeciesspecific primers [19].

An alternative for the aforementioned molecular identification can be the sequencing of the $16 \mathrm{~S}$ rRNA, in combination with $g y r B$ gene sequencing generally regarded as a golden standard [16, 19, 20]. Zhou's test confirms that by having examined 181 isolates the similarities with the sequences in GenBank reached over 99\% and 96\% [20]. According to Zhou et al., however, the sequencing of the $g y r B$ gene can be more practical and more precise in identifying VGS strains than other methods [20].

The basis of species identification appears to be the phenotypical tests used by many researchers; they confirm the fact that they belong to a given species and that the findings of MALDI-TOF MS are correct. Molecular techniques allow 
TABLE 2: Virulence factors and morbidity against human of group C streptococci.

\begin{tabular}{|c|c|c|c|c|}
\hline Species & Virulence factors & Disease & Origin & References \\
\hline S. equi subs. equi & Protein & Septic arthritis, meningitis & Animals, human & {$[1,6,11]$} \\
\hline S. equi subs. zooepidemicus & Surface-exposed protein (Szp) & $\begin{array}{l}\text { Meningitis, bacteremia, } \\
\text { endocarditis }\end{array}$ & $\begin{array}{l}\text { Unpasteurized milk; } \\
\text { animals }\end{array}$ & {$[6,9,11]$} \\
\hline $\begin{array}{l}\text { S. dysgalactiae subs. } \\
\text { equisimilis }\end{array}$ & $\begin{array}{c}\text { Surface-exposed protein (Szp) } \\
\text { emm gene homologs, } \\
\text { streptokinase, streptolysin O } \\
\text { and S, M protein, hemolysin, } \\
\text { C5a peptidase, glyceraldehyde } \\
\text { 3-phosphate dehydrogenase } \\
\text { (GAPDH), hyaluronidase }\end{array}$ & $\begin{array}{l}\text { Pharyngitis, pneumonia, } \\
\text { endocarditis, meningitis, acute } \\
\text { glomerulonephritis, skin and soft } \\
\text { tissue infections, septic arthritis, } \\
\text { osteomyelitis, endometritis, } \\
\text { streptococcal toxic shock-like } \\
\text { syndrome, necrotizing fasciitis, } \\
\text { cellulitis, septic arthritis, } \\
\text { bacteremia, RF } \text { RHD }^{\mathrm{a}}\end{array}$ & $\begin{array}{l}\text { Unpasteurized dairy } \\
\text { products, animals, } \\
\text { human }\end{array}$ & $\begin{array}{c}{[2,5,7,9-} \\
11,13]\end{array}$ \\
\hline
\end{tabular}

${ }^{\mathrm{a}} \mathrm{RF}$ : rheumatic fever.

${ }^{b}$ RHD: rheumatic heart disease.

us also to detect or confirm the presence of some features which cannot be examined by means of the phenotypical methods of biochemical tests. These two groups of methods are inseparably linked, and in order to precisely examine the group C streptococci one should combine the phenotypical methods with molecular techniques.

New methods of identification based on protein typing or genome typing are barely available in Polish laboratories; therefore latex tests prevail. Probably, such a situation is also typical of other East and Central European countries, or in the developing countries. Therefore literature contains mainly the findings from the highly developed countries.

\section{Virulence Factors}

The virulence factor in some species of GCS most frequently mentioned is the $\mathrm{M}$ protein, the protein that also belongs to group A streptococci $[2,5,7,10,12,14,21,22]$. This antiphagocytic, fibrillar, and surface-exposed $M$ protein [14, $15]$ is encoded by the emm gene [12]. Bisno's tests have shown that M protein can mainly be found in S. dysgalactiae subs. equisimilis isolates isolated from patients suffering from acute pharyngitis [15]. There are over 80 well-known $\mathrm{M}$ protein serotypes of group A streptococci [15]. In comparative research between $M$ proteins of GAS and $M$ proteins of GCS isolates SDSE was examined from people and it was found that the structures of $M$ proteins of GAS were highly homological to $\mathrm{M}$ proteins of GCS in the $\mathrm{C}$ repeat region but the $\mathrm{N}$ terminus was unique [15].

It seems that $M$ protein plays an important role in rheumatic fever (RF) $[5,15]$. McDonald and Bramhachari have noticed that GCS isolates more often than GAS colonize the throats of Aboriginal population suffering from $\mathrm{RF}$ incidence and rheumatic heart disease (RHD) [5, 21]. Dinkla's work seems to confirm that $\mathrm{M}$ and $\mathrm{M}$-like proteins of group $\mathrm{C}$ streptococci take part in the pathogenesis of rheumatic fever in some geographic regions [18]. All of the 70 isolates $S$. dysgalactiae subs. equisimilis belonged to GCS/GGS; 27 of them had the ability to bind collagen IV and these were subjected to further analysis [18]. Out of 27 SDSE strains containing the $\mathrm{emm}$ gene, coding M proteins, 4 had the fog gene coding M-like fibrinogen binding protein of GGS (FOG) [18]. These findings are confirmed in Haidan's and Rantala's reports where GCS/GGS isolates were found, and the isolates that have the fibronectin-binding protein property can aggregate human platelets and can toxically affect the endothelial cells [13, 23]. Dinkla's research shows that 27 SDSE strains owe their ability to bind collagen IV to the hyaluronic acid capsule [18]. Furthermore McDonald and Nataneli describe the capacities of GCS for cross reaction with cardiac epitopes and their superantigenic features [21, 22].

Aside from M protein, some species of GCS (Table 2) can produce a surface-exposed protein (Szp), streptokinase, streptolysin O or streptolysin S [2, 10], and hemolysins [7]. Surface-exposed protein is produced by $S$. equi subs. zooepidemicus [11]. Chandnani indicates the SDSE strain to produce streptokinase and streptolysin $\mathrm{O}$ and shows theories about the pathogenesis of rheumatic fever [2]. There is a direct cytotoxic influence of streptolysin $\mathrm{O}$ on the cardiac cells and other organs or there are antigenic determinants between the components of an organism and some specific tissues, such as the brain or heart, that is a result of immunologic cross reactivity [2]. Following a comparative analysis of the GAS genome with the SDSE genome, Silva and Watanabe confirm that they contain related virulence factors $[10,14]$.

Besides the aforementioned virulence factors SDSE isolates have C5a peptidase, glyceraldehyde 3-phosphate dehydrogenase, and hyaluronidase [10]. There are also several important GAS virulence factors, which are not found in the SDSE genome, for example, cysteine protease, and a superantigen homolog [14]. SDSE enzymes, matrix metalloproteinases, and plasminogen binding GAPDH enzyme contribute to dissemination in the body and proliferation. An analysis of the genome of SDSE 167 strain has shown that it excretes lyases decomposing carbohydrates of the host, while at the same time this genome metabolizes carbohydrates in the Entner-Doudoroff pathway; this region contains the enzyme metabolizing polysaccharides, which may contribute 
to a higher virulence of SDSE 167 strain [14]. Watanabe et al. claim that SDSE 167 strain is the most virulent human strain [14].

\section{Pathogenicity}

Among the diseases caused by the species of GCS the dominating ones are pharyngitis, meningitis, and endocarditis (Table 2). According to Danish data (1999-2002), GCS infections take the last place among streptococcal infections, but they constitute as many as $6 \%$ of all streptococcal infections [3]. This has also been confirmed by the Norwegian observations of 1999-2013, where out of 512 invasive infections GCS was the cause of 24 cases (4,7\%) [12]. In both reports, the risk of infection increases with the patient's age $[3,12]$. Among other factors that predispose one to infections are diabetes, tumors, cardiac conditions, and alcohol abuse [3], whereas Rantala adds immunosuppressive therapy, multiple morbidities, and skin lesions as potential predisposing factors [13].

The most frequent clinical form of invasive virulent species of GCS infections are infections of bones, joints, and blood infections, and the noninvasive infections of the upper respiratory tracts [12]. The mortality rate during the first 30 days of the disease, in the Danish research, equaled $21 \%$, whereas in the Norwegian research it equaled $9 \%$, and it increased with age and with respect to the kind of disease $[3,12]$. Ekelund et al. reports that the highest rate concerned patients with GAS (23\%) and GCS infections (21\%) [3]; in Oppegaard et al. the mortality rate in GAS infections was $13 \%$ [12]. Both Ekelund et al. and Oppegaard et al. list similar diseases: skin infections (erysipelas, cellulitis), necrotizing fasciitis, and toxic shock syndrome $[3,12]$.

Rantala presents two cases of elderly patients with recurring bacteremia caused by the most frequent GCS isolates, SDSE, and a broad range of diseases caused by the following subspecies: pharyngitis, tonsillitis, skin infection, soft tissue infection, wound infections, erysipelas, cellulitis, necrotizing fasciitis, streptococcal toxic shock-like syndrome, pneumonia, septic arthritis, osteomyelitis, meningitis, endocarditis, and bacteremia [13].

The findings of the British Health Protection Agency and Watanabe are alarming. They have shown a $100 \%$ increase of bacteremia between 2006 and 2012 and an increase of SDSE infections in Asia, Europe, and the USA [12, 14]. Of particular interest are the reports about the relations between GCS with the pathogenesis of rheumatic fever in Australia and India [5, 21, 23] and first documented zoonotic diseases $[6,8,9,22,24]$.

As has already been mentioned, though RF pathogenesis is not clear only in the upper respiratory tract infection, GAS may directly lead to rheumatic fever. Haidan's studies have shown that in Australia higher indicators of isolation of GCS have been found as well as the highest rate of RF-dependent morbidity in the world [23], a fact which, as researchers say, may be related to high RF and RHD morbidity among Aboriginal communities of Australia [5, 21].
Nataneli et al. have described an American case of poststreptococcal syndrome uveitis (PSU), which is the first documented case of complications following pharyngitis and caused by GCS infection [22]. The patient was a 24-yearold man with HLA-B27 positive genotype (this genotype predisposing an individual also to poststreptococcal reactive arthritis). Morsch, however, describes the case of a 72-yearold man, suffering from tophaceous gout, in whom olecranon bursitis developed as the result of a GCS infection [24]. The author notes that until now circa 15 cases of septic arthritis (GCS) have been documented, but none have been related to bursitis [24].

In publications one can find some data concerning zoonotic diseases. The first report describes the case of a 13year-old boy who lived on a horse farm; he became ill with meningitis caused by $S$. equi subs. equi [6]. The second case is of a 13-year-old girl suffering from meningitis caused by S. equi subs. zooepidemicus [9]. A day before her illness she took part in horse races [9]. A description of GCS-dependent meningitis with cavernous sinus in an 18-year-old man is worth mentioning [8]. Virulent species of GCS can also cause, among other things, impetigo, epiglottitis, and polyarthritis $[5,6,21]$.

\section{Conclusions}

(1) Virulent species of group C streptococci, which cause infection in people, are most often animals' pathogens and belong to bacteria that are difficult to be identified. Due to the common origin of GCS and GAS the method of identification is to combine phenotypical and molecular techniques.

(2) The study of the epidemiology of human GCS infections is possible owing to the application of modern NGS (Next Generation Sequencing) methods in routine investigations.

(3) Some species of GCS, chiefly SDSE, have many virulence factors found in GAS like M protein, streptokinase, and streptolysins, but there are some that are missing like cysteine protease. There are assumptions that virulent species of GCS can be related to the pathogenesis of rheumatic fever and rheumatic heart disease, especially among Aboriginal population of Australia.

(4) The main origins of virulent species of GCS are unpasteurized dairy products, animals, and humans.

(5) The most frequent diseases are pharyngitis and meningitis. The most frequent GCS isolates from human infection is SDSE.

(6) The predisposing factors are, among other things, age and chronic diseases. The range of GCS-dependent diseases is broad, including infections related to the respiratory system, skin infections and soft tissue infections, necrotizing fasciitis, streptococcal toxic shock syndrome, septic arthritis, osteomyelitis, endocarditis, and bacteremia. 


\section{Conflicts of Interest}

The authors declare that there are no conflicts of interest regarding the publication of this paper.

\section{References}

[1] C. A. Guillén Astete, N. Sánchez Gómez, and M. Luque Alarcón, "Septic Arthritis by Streptococcus equi," Reumatología Clínica (English Edition), vol. 12, no. 4, pp. 237-238, 2016.

[2] H. K. Chandnani, R. Jain, and P. Patamasucon, "Group C streptococcus causing rheumatic heart disease in a child," Journal of Emergency Medicine, vol. 49, no. 1, pp. 12-14, 2015.

[3] K. Ekelund, P. Skinhøj, J. Madsen, and H. B. Konradsen, "Invasive group A, B, C and G Streptococcal infections in Denmark 1999-2002: epidemiological and clinical aspects," Clinical Microbiology and Infection, vol. 11, no. 7, pp. 569-576, 2005.

[4] R. C. Lancefield, "A serological differentiation of human and other groups of hemolytic streptococci," Journal of Experimental Medicine, vol. 57, no. 4, pp. 571-595, 1933.

[5] P. V. Bramhachari, S. Y. Kaul, D. J. McMillan, M. S. Shaila, M. G. Karmarkar, and K. S. Sriprakash, "Disease burden due to Streptococcus dysgalactiae subsp. equisimilis (group G and C streptococcus) is higher than that due to Streptococcus pyogenes among Mumbai school children," Journal of Medical Microbiology, vol. 59, no. 2, pp. 220-223, 2010.

[6] S. Elsayed, O. Hammerberg, V. Massey, and Z. Hussain, “Streptococcus equi subspecies equi (Lancefield group C) meningitis in a child," Clinical Microbiology and Infection, vol. 9, no. 8, pp. 869-872, 2003.

[7] T. Zaoutis, M. Attia, R. Gross, and J. Klein, "The role of group $\mathrm{C}$ and group G streptococci in acute pharyngitis in children," Clinical Microbiology and Infection, vol. 10, no. 1, pp. 37-40, 2004.

[8] M. Clarke, H. Eenuh, J. Ssaverimuttu, and J. Nfonoyim, "Streptococcus group C meningitis with cavernous sinus thrombosis," Infection and Drug Resistance, vol. 6, pp. 79-81, 2013.

[9] S. S. Shah, R. P. Matthews, and C. Cohen, "Group C streptococcal meningitis: case report and review of the literature," Pediatric Infectious Disease Journal, vol. 20, no. 4, pp. 445-448, 2001.

[10] L. G. Silva, G. L. Genteluci, M. C. de Mattos, T. Glatthardt, A. M. Sà Figueiredo, and B. T. Ferreira-Carvalho, "Group C Streptococcus dysgalactiae subsp. equisimilis in south-east Brazil: genetic diversity, resistance profile and the first report of human and equine isolates belonging to the same multilocus sequence typing lineage," Journal of Medical Microbiology, vol. 64, pp. 551-558, 2015.

[11] R. Facklam, "What happened to the streptococci: overview of taxonomic and nomenclature changes," Clinical Microbiology Reviews, vol. 15, no. 4, pp. 613-630, 2002.

[12] O. Oppegaard, H. Mylvaganam, and B. R. Kittang, "Betahaemolytic group A, C and G streptococcal infections in Western Norway: a 15-year retrospective survey," Clinical Microbiology and Infection, vol. 21, no. 2, pp. 171-178, 2015.

[13] S. Rantala and S. Tuohinen, "Two cases of cardiac device-related endocarditis due to Streptococcus dysgalactiae subsp. equisimilis (group C or G streptococci)," BMC Infectious Diseases, vol. 14, no. 1, pp. 174-178, 2014.

[14] S. Watanabe, T. Kirikae, and T. Miyoshi-Akiyama, "Complete genome sequence of Streptococcus dysgalactiae subsp. equisimilis 167 carrying lancefield group $\mathrm{C}$ antigen and comparative genomics of S. dysgalactiae subsp. equisimilis strains," Genome Biology and Evolution, vol. 5, no. 9, pp. 1644-1651, 2013.

[15] A. L. Bisno, C. M. Collins, and J. C. Turner, "M proteins of group C streptococci isolated from patients with acute pharyngitis," Journal of Clinical Microbiology, vol. 34, no. 10, pp. 2511-2515, 1996.

[16] S. Angeletti, G. Dicuonzo, A. Avola et al., "Viridans group streptococci clinical isolates: MALDI-TOF mass spectrometry versus gene sequence-based identification," PLOS ONE, vol. 10, no. 3, Article ID e0120502, 2015.

[17] M. W. Cunningham, "Pathogenesis of group a streptococcal infections," Clinical Microbiology Reviews, vol. 13, no. 3, pp. 470511, 2000.

[18] K. Dinkla, D. P. Nitsche-Schmitz, V. Barroso et al., "Identification of a streptococcal octapeptide motif involved in acute rheumatic fever," Journal of Biological Chemistry, vol. 282, no. 26, pp. 18686-18693, 2007.

[19] M. Ciszewski, K. Zegarski, and E. M. Szewczyk, "Streptococcus dysgalactiae subsp. equisimilis isolated from infections in dogs and humans: are current subspecies identification criteria accurate?” Current Microbiology, vol. 73, no. 5, pp. 684-688, 2016.

[20] M. Zhou, Q. Yang, T. Kudinha et al., "Using matrix-assisted laser desorption ionization-time of flight (MALDI-TOF) complemented with selected $16 \mathrm{~S}$ rRNA and gyrB genes sequencing to practically identify clinical important viridans group streptococci (VGS)," Frontiers in Microbiology, vol. 7, article 1328, 2016.

[21] M. McDonald, B. J. Currie, and J. R. Carapetis, "Acute rheumatic fever: a chink in the chain that links the heart to the throat?" Lancet Infectious Diseases, vol. 4, no. 4, pp. 240-245, 2004.

[22] N. Nataneli, Z. P. Aguilera, P. S. Rosenbaum, T. Goldstein, and M. Mayers, "Poststreptococcal keratouveitis associated with group C streptococcus pharyngitis," Clinical Ophthalmology, vol. 5, no. 1, pp. 1257-1259, 2011.

[23] A. Haidan, S. R. Talay, M. Rohde, K. S. Sriprakash, B. J. Currie, and G. S. Chhatwal, "Pharyngeal carriage of group C and group $\mathrm{G}$ streptococci and acute rheumatic fever in an Aboriginal population," The Lancet, vol. 356, no. 9236, pp. 1167-1169, 2000.

[24] C. Predebon Morsch, F. Batistella, and H. Luiz Staub, “Olecranon bursitis caused by group $\mathrm{C}$ streptococcus in a patient with tophaceous gout," Brazilian Journal of Infectious Diseases, vol. 17, no. 1, pp. 116-117, 2013. 


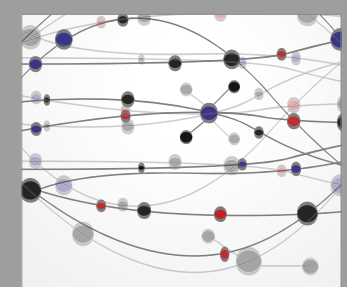

The Scientific World Journal
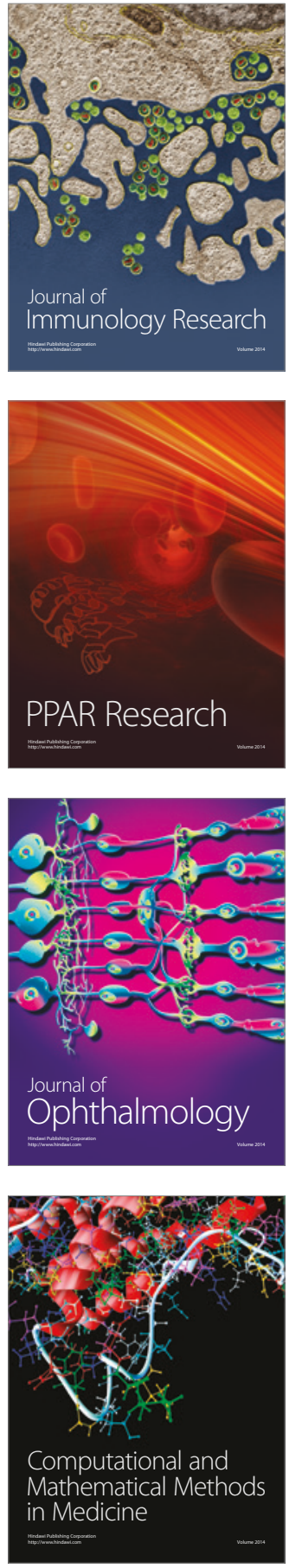

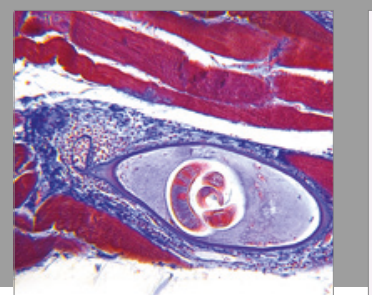

Gastroenterology Research and Practice
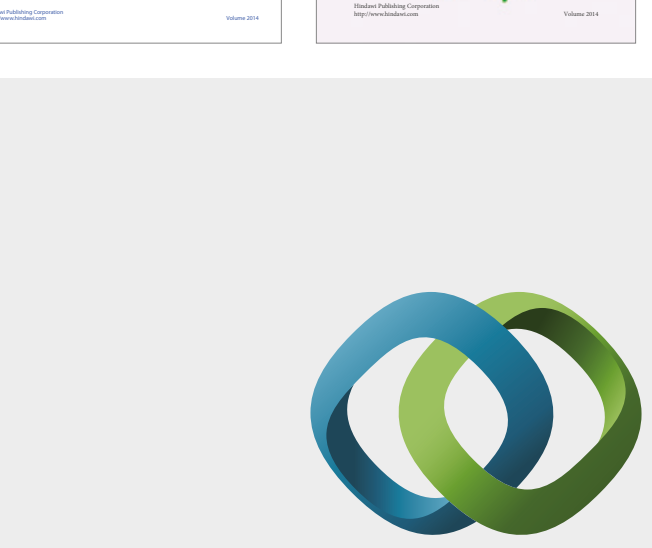

\section{Hindawi}

Submit your manuscripts at

https://www.hindawi.com
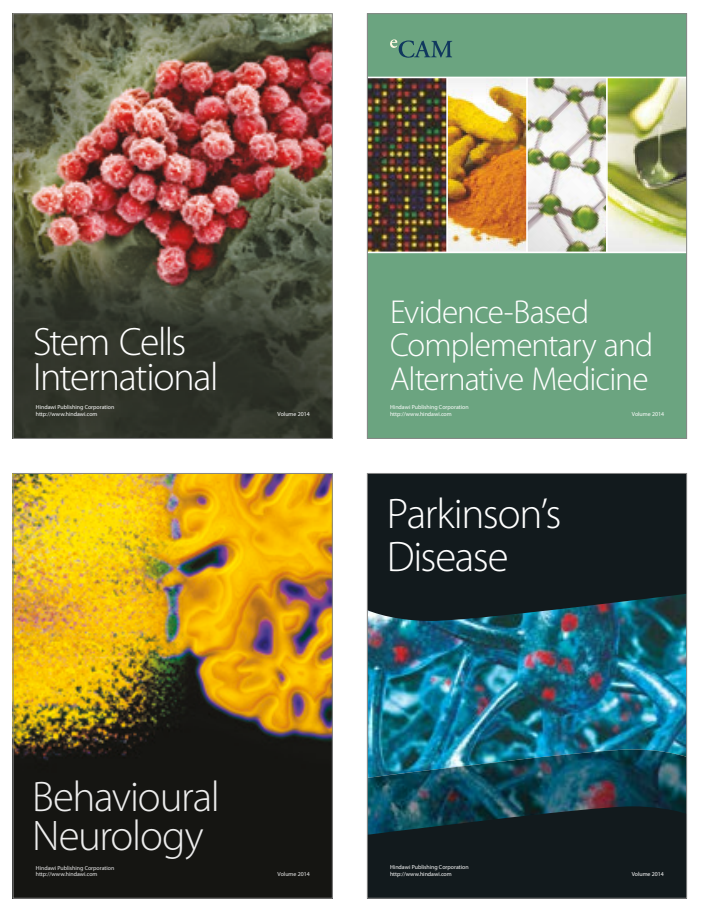
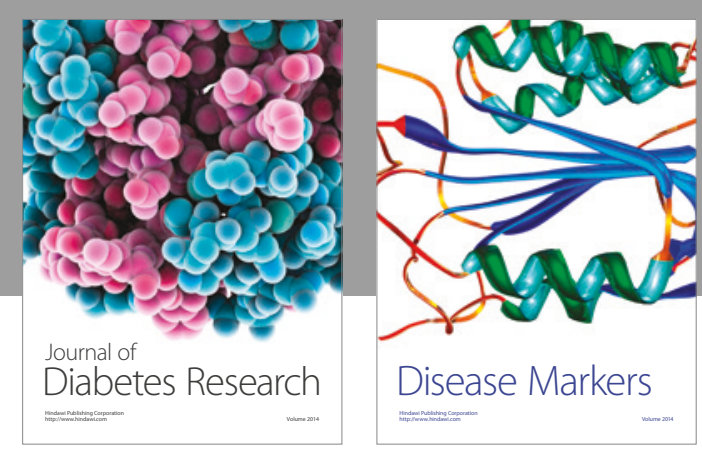

Disease Markers
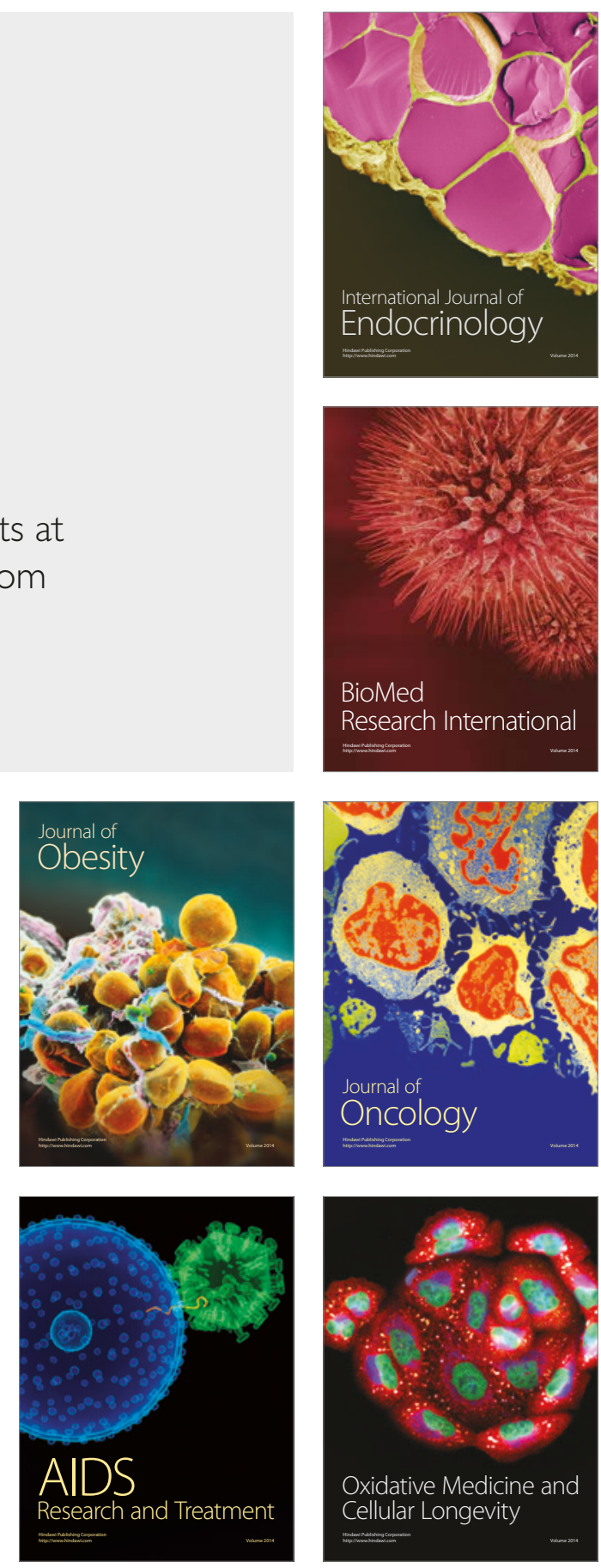